\title{
From lattice QCD to in-medium heavy-quark interactions via deep learning
}

\author{
Shuzhe Shi ${ }^{1, *}$, Kai Zhou ${ }^{2}$, Jiaxing Zhao ${ }^{3}$, Swagato Mukherjee ${ }^{4}$, and Pengfei Zhuang ${ }^{3}$ \\ ${ }^{1}$ Department of Physics, McGill University, Montreal, Quebec H3A 2T8, Canada. \\ ${ }^{2}$ Frankfurt Institute for Advanced Studies, Ruth Moufang Strasse 1, D-60438, Frankfurt am Main, \\ Germany. \\ ${ }^{3}$ Department of Physics, Tsinghua University, Beijing 100084, China. \\ ${ }^{4}$ Physics Department, Brookhaven National Laboratory, Upton, New York 11973, USA.
}

\begin{abstract}
Bottomonium states are key probes for experimental studies of the quark-gluon plasma (QGP) created in high-energy nuclear collisions. Theoretical models of bottomonium productions in high-energy nuclear collisions rely on the in-medium interactions between the bottom and antibottom quarks, which can be characterized by real $\left(V_{R}(T, r)\right)$ and imaginary $\left(V_{I}(T, r)\right)$ potentials, as functions of temperature and spatial separation. Recently, the masses and thermal widths of up to $3 S$ and $2 P$ bottomonium states in QGP were calculated using lattice quantum chromodynamics (LQCD). Starting from these LQCD results and through a novel application of deep neural network (DNN), here, we obtain model-independent results for $V_{R}(T, r)$ and $V_{I}(T, r)$. The temperature dependence of $V_{R}(T, r)$ was found to be very mild between $T \approx 0-330 \mathrm{MeV}$. Meanwhile, $V_{I}(T, r)$ shows rapid increase with $T$ and $r$, which is much larger than the perturbation theory based expectations.
\end{abstract}

In-medium modifications of quarkonium states are sensitive probes of the QGP produced in high energy nuclear collisions [1-13]. Sequential suppression patterns among the $\Upsilon(1 S)$, $\Upsilon(2 S)$ and $\Upsilon(3 S)$ states have been observed in heavy-ion collision experiments [14-17]. Theoretical understanding of these experimental observations rely on effective field theories (EFT), which naturally lead to an open quantum system based treatment of both open and hidden bottom states in QGP (for a recent review, see [12]). If interactions between the colorsinglet and color-octet states are neglected then the pNRQCD reduces to a theoretical description of quarkonia solely based on a potential between the heavy quark and antiquark. A potential based description allows studies of quarkonia by employing Schrödinger-type equations [18-21]. One-loop hard thermal loop (HTL) perturbative QCD calculations [22, 23], and later on pNRQCD calculations [24, 25], show that at high temperatures heavy quark potential becomes complex with a nonvanishing imaginary part. However, it is difficult to provide satisfactory descriptions of bound states arising out of strong interactions solely using perturbative expansions and a nonperturbative treatment, such as the LQCD, is called for. In the static limit, the heavy quark potential can be extracted from the spectral functions of the thermal Wilson loop using nonperturbative LQCD calculations [26-29]. To extract the heavy quark potential from the Euclidean-time Wilson loop spectral functions, these LQCD calculations rely on weak-coupling motivated functional forms of the spectral functions. On the

\footnotetext{
*e-mail: shuzhe.shi@mcgill.ca
} 
other hand, recent LQCD studies have led to quantification of the masses, thermal widths, and Bethe-Salpeter amplitudes (BSA) of up to $3 S$ and $2 P$ bottomonium states in QGP [30-32]. As we shall see later, one-loop HTL motivated functional forms of $V_{R}(T, r)$ and $V_{I}(T, r)$ are not compatible with the recent LQCD results. This observation calls for a model-independent nonperturbative extraction of the in-medium heavy quark potential.

In our recent work [33], we have developed a model-independent DNN-based method and determine the $r$ and $T$-dependence of the in-medium heavy quark potential starting from the LQCD results [31] for the masses and thermal widths of up to $3 S$ and $2 P$ bottomonium states at various temperatures. The underlying idea is as follows: At a fixed $T$, various bottomonium states differ in sizes and their wavefunctions probe different distances. Knowledge of the masses and thermal widths of multiple bottomonium states, thereby, provide constraints on not only the strength of the real and imaginary parts of the bottom-antibottom interactions in QGP but also its $r$-dependence. Thus, LQCD results for the masses and thermal widths of multiple bottomonium states at different temperature can be used to extract $V_{R}(T, r)$ and $V_{I}(T, r)$ and, presently, DNN is probably the best tool achieve this in an unbiased fashion. According to the universal approximation theorem [34, 35], DNN can generally provide an unbiased, yet flexible enough, parameterization to approximate arbitrary functional relations. We exploited the DNNs to represent the real and imaginary potentials,

$$
V_{R}(T, r)=V_{R, \mathrm{DNN}}\left(\boldsymbol{b}_{R}, \boldsymbol{W}_{R} ; T, r\right), \quad V_{I}(T, r)=V_{I, \mathrm{DNN}}\left(\boldsymbol{b}_{I}, \boldsymbol{W}_{I} ; T, r\right),
$$

where $\boldsymbol{b}$ and $\boldsymbol{W}$ - called bias and weight, respectively - are the DNN parameters to be determined by fitting the LQCD masses and thermal widths [31]. In this Proceeding, we will briefly summarize our method and results. We will further discuss the possible interpretation and phenomenological consequence, as the complement of [33].

In typical machine-learning problems, one usually knows some direct informations of the target function - e.g., its value at discrete points - and is able to tune the DNN parameters by directly compare its output to the known knowledge. In our network, however, we cannot compare Eq. (1) to the LQCD masses and thermal widths and fix the parameters. There is an extra step to map the DNN output (potential) to observables (masses and thermal widths) the reduced complex-valued two-body time-independent Schrödinger equation, ${ }^{1}$

$$
-\frac{\nabla^{2}}{m_{b}} \psi_{n}+\left[V_{R}(T, r)+i \cdot V_{I}(T, r)\right] \psi_{n}=E_{n} \psi_{n}
$$

where the wavefunction $\psi_{n}$ and the energy eigenvalues $E_{n}$ for in-medium bottomonia are complex-valued. Here, $V_{I}(T=0, r)=0, \operatorname{Re}\left[E_{n}\right]=m_{n}-2 m_{b}$ and $\operatorname{Im}\left[E_{n}\right]=-\Gamma_{n}$, where $m_{n}$ and $\Gamma_{n}$ are the mass and thermal width of the $n^{\text {th }}$ bottomonium state, respectively. We solved Eq. (2) with potential provided by DNN (1) and obtain the masses and thermal widths. The DNN parameters $\left(b_{i}^{(l)}\right.$ and $\left.W_{i j}^{(l)}\right)$ are tuned by minimizing the cost function

$$
J=\frac{\lambda}{2} \sum_{l, i}\left(b_{i}^{(l)}\right)^{2}+\frac{\lambda}{2} \sum_{l, i, j}\left(W_{i j}^{(l)}\right)^{2}+\frac{1}{2} \sum_{T, n}\left(\frac{m_{T, n}-m_{T, n}^{\mathrm{LQCD}}}{\delta m_{T, n}^{\mathrm{LQCD}}}\right)^{2}+\left(\frac{\Gamma_{T, n}-\Gamma_{T, n}^{\mathrm{LQCD}}}{\delta \Gamma_{T, n}^{\mathrm{LQCD}}}\right)^{2},
$$

Here, the $\propto \lambda$ terms are regularizers in DNN to avoid over-fitting. The summation runs over six temperature points, $T \in\{0,151,173,199,251,334\} \mathrm{MeV}$, and five bottomonium states, $n \in\{1 \mathrm{~S}, 2 \mathrm{~S}, 3 \mathrm{~S}, 1 \mathrm{P}, 2 \mathrm{P}\}$ and the LQCD values were taken from Ref. [31]. We used gradient descent with Back-Propagation optimization technique, which is based on the derivatives of

\footnotetext{
${ }^{1}$ By taking the Cornell potential, we can well reproduce the vacuum masses of up to $3 S$ and $2 P$ bottomonium states [36] and the corresponding vacuum BSA obtained from LQCD calculation [32], (see [33] for more details).
} 


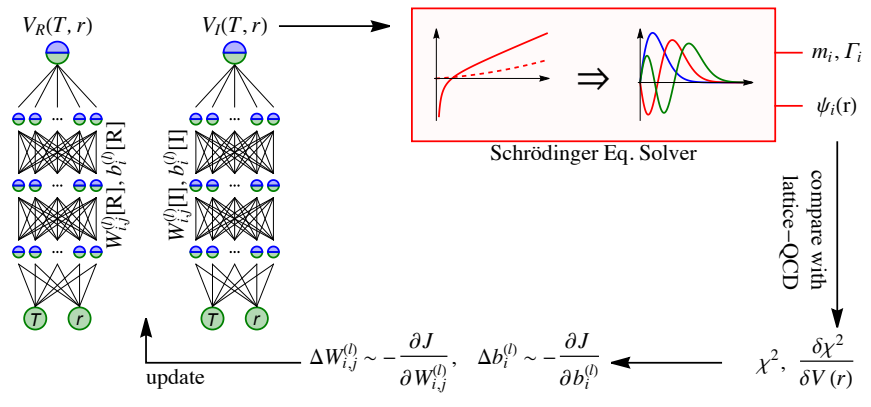

Figure 1. Flow chart of the potential reconstruction scheme - using generalized back-propagation to optimize parameters in the deep neural networks coupled with a Schrödinger equation.

the cost function with respect to the network parameters. We overcame the challenge of gradients evaluation of such implicit functions through perturbative solution of the Schrödinger equation with respect to small change of $V(T, r)$. Moreover, we invoked Bayesian inference for uncertainty quantification, whereby the posterior distribution of the network parameters was evaluated. In Fig. 1 we show the flow chart of our methodology of the potential reconstruction with DNNs coupled to a Schrödinger equation. To the best of our knowledge, the current method is developed for the first time here. More details on the method is provided in [33], along with a closure test to justify our methodology and assess its reliability.

Results and Discussions - We begin with pointing out the inadequacy of weak-coupling motivated functional form of the potential to consistently describe the LQCD masses and thermal widths. We chose the functional form proposed in Ref. [37], which incorporates one-loop HTL based functional forms of $V_{I}$ and of color-electric screening, in addition to a vacuum potential satisfying Gauss's law. Taking this functional form for the potential, we fix $\alpha, \sigma$, and $B$ by their vacuum values, and tune $\mu_{D}$ at different temperatures to fit the finitetemperature bottomonia masses and widths. As shown by the open squares in Fig. 2, one-loop HTL motivated functional form of $V_{I}$ and color-electric screening in $V_{R}$ fail to simultaneously reproduce the LQCD results for the mass shifts and the thermal widths of bottomonium. Even if allowing an extra magnification factor for $V_{I}$, one would still miss the state-dependence of the thermal width (see open diamonds which take magnification factor to be four). The failure of the only known analytic form to describe the LQCD results necessitates a modelindependent extraction of $V(T, r)$ using an adequate unbiased parameterization. We devised
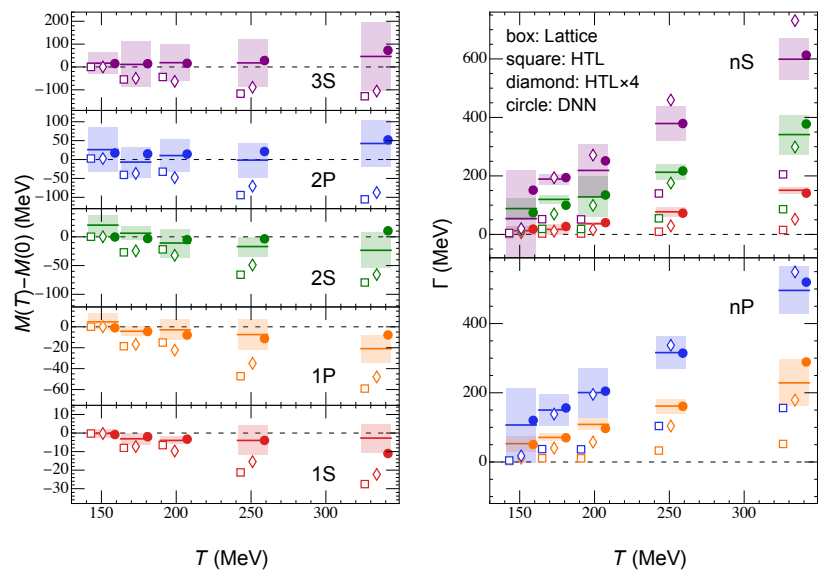

Figure 2. In-medium mass shifts (left) and the thermal widths (right) of different bottomonium states obtained from fits to LQCD results [31] (lines and shaded bands) using HTL functional forms [37] (open symbols) and DNN based optimization (filled circle). The points are shifted horizontally for better visualization. $\Upsilon(1 S)$, $\chi_{b_{0}}(1 P), \Upsilon(2 S), \chi_{b_{0}}(2 P)$ and $\Upsilon(3 S)$ states are represented by red, orange, green, blue, and purple symbols, respectively. 
the above outlined method by coupling Schrödinger equation with DNNs and achieved good agreement with the LQCD results [31] (see solid symbols in Fig. 2).

The $T$ - and $r$-dependence of the extracted real and imaginary potentials are shown in Fig. 3. We see signs that with increasing temperature $V_{R}(T, r)$ becomes flatter at large $r$, as expected from color screening effect. However, the temperature dependence of $V_{R}(T, r)$ is very mild between $T \approx 150-330 \mathrm{MeV}$, and closely approximating its vacuum counterpart. In the same temperature range, we observed that $V_{I}(T, r)$ monotonically increases both with temperature and distance. Whereas $V_{I}$ varies a lot as temperature changes, the scaled imaginary potential, $V_{I} / T$ as function of $r \times T$, is insensitive to the change of temperature, (see Right panel of Fig. 3).
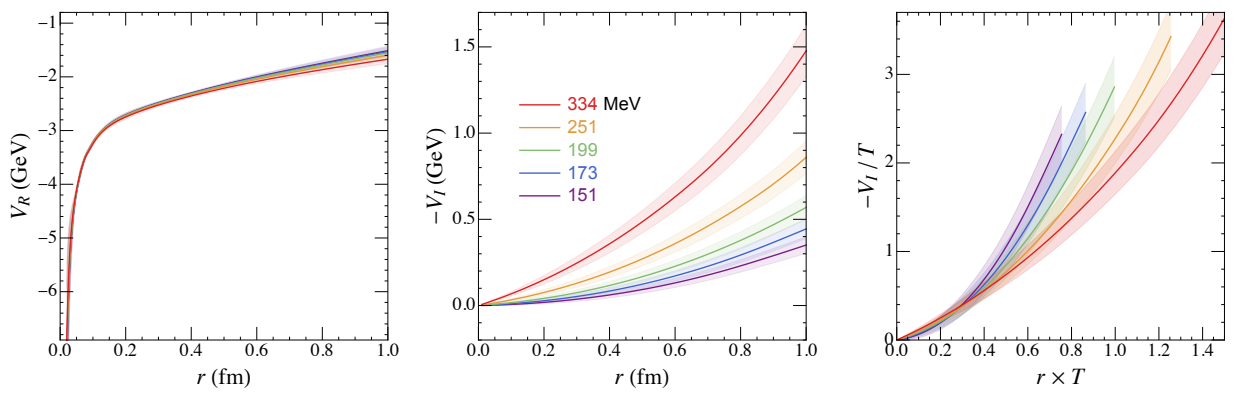

Figure 3. The DNN reconstructed real (Left) and imaginary (Middle) parts of the heavy quark potential at temperatures $T=151$ (purple), 173(blue), 199(green), 251(orange), and $334 \mathrm{MeV}$ (red). The uncertainty bands represent the $68 \%(1 \sigma)$ confident region. Right panel is the same as Middle, but for $x$ - and $y$-axis scaled by temperature $T$.

The heavy quark potential obtained here is based on LQCD calculations of bottomonium state using $2+1$ flavor dynamical gauge field background with nearly physical values of up, down, and strange quark masses. Whereas the extant LQCD calculations of $V_{R}$ and $V_{I}$ are for infinitely heavy static quarks and mostly in pure SU(3) gauge theory without dynamical quarks [26-29]. Direct quantitative comparison, thus, is difficult. However, our results for the heavy quark potential is qualitatively different from the weak-coupling motivated extractions of the static quark potentials [26-29]. Unlike the previous studies, the $V_{R}$ obtained in this work show very little signs of color-electric Debye screening for $r \lesssim 1 \mathrm{fm}$ for the entire temperature range $T \in[0,334] \mathrm{MeV}$. The $V_{I}$ here is much larger in magnitude and increases more rapidly both with $T$ and $r$ than the one-loop HTL motivated extractions. On the other hand, it is reassuring that the preliminary results on the static quark potential from very recent LQCD calculations and without using the one-loop HTL motivated forms are quite similar to the potential obtained here [38, 39].

Based on our results, one might speculate that, for phenomenologically relevant temperatures $T \lesssim 330 \mathrm{MeV}$, bottomonia are approximately vacuum-like excitation but very short lifetime that are inversely proportional to their large thermal widths. This is different from the conventional picture of the bottomonium in-medium behavior. In the traditional picture, both the finite temperature free-energy and interaction potential becomes flat at large distance. As the temperature increases, the platform decreases in height, thus the binding energy of the boundstate decreases, while its average size increase. At a certain temperature, $T_{m}$, the binding energy approaches to zero, and the average size diverges $[18,19,40] . T_{m}$ is referred to as the melting temperature, above which a boundstate no longer exists. Similar 
behaviors are observed for potentials with non-vanishing imaginary part [22, 23, 37, 41, 42]. According to the recent lattice QCD calculation for static quark potential [37], the $\Upsilon(3 S)$ state melts at $T \sim 0.2 \mathrm{GeV}$, while $\Upsilon(2 \mathrm{~S})$ disappears at $T \gtrsim 0.35 \mathrm{GeV}$. In our new picture, on the contrary, all the five bottomonium states can survive up to $T=334 \mathrm{MeV}$, and their masses are insensitive to the temperature. On the other hand, the imaginary potential induced thermal widths, which characterize the dynamical dissociation and increase with temperature, are significantly larger than previous results [37]. The inverse of thermal width can be interpreted as lifetime of state at finite temperature. For instance, at $T=334 \mathrm{MeV}$, we find $\Gamma(1 \mathrm{~S}) \sim 0.15 \mathrm{GeV}, \Gamma(2 \mathrm{~S}) \sim 0.3 \mathrm{GeV}$, and $\Gamma(3 \mathrm{~S}) \sim 0.6 \mathrm{GeV}$, correspond to lifetime $1.3 \mathrm{fm}$, $0.7 \mathrm{fm}$, and $0.3 \mathrm{fm}$, respectively. In such a new continuous dissociation picture, the enhancement of the thermal width compensates the vanishing of the melting effect in the thermal dissociation.

This is important for studying the Quarkonium dynamics in the rapidly expanding QGP in heavy ion collisions. The suppression of the Quarkonia production rate in relativistic heavyion collisions is caused by both the color-screening melting and the dynamical dissociation, the new picture suggests a reduction of the former and an enlargement of the latter. Noting that such two effects exhibit different temperature dependencies, phenomenological consequence might be expected in the experimental measurements of azimuthal angle distribution of the production rate, especially for the excitations.

Conclusion - In this work, a bias-free in-medium heavy quark potential with DNN representation is determined from the recently obtained LQCD results [31] for the masses and thermal widths of up to $3 S$ and $2 P$ bottomonium states in QGP. By coupling Schrödinger equation to DNN, we introduced a novel method for unbiased extractions of the real and imaginary parts of the heavy quark potential, and invoked Bayesian inference to quantify the potential uncertainties in a non-local fashion. We obtained model-independent results for $V_{R}(T, r)$ and $V_{I}(T, r)$ for $r \lesssim 1 \mathrm{fm}$ and $T \lesssim 330 \mathrm{MeV}$. The $V_{R}(T, r)$ has very mild $T$ dependence and closely resembles the vacuum potential. On the other hand, $V_{I}(T, r)$ is large and rises rapidly with $T$ and $r$. These results are qualitatively different from the static quark potential obtained using one-loop HTL perturbative calculations. It would be very interesting to see the phenomenological consequences [13] of this heavy quark potential, model-independently extracted from the non-perturbative LQCD calculations.

The authors thank Min He, Matthew Heffernan, Rasmus Larsen, Simon Mak, Peter Petreczky, Ralf Rapp, Alexander Rothkopf, and Nan Su for many insightful discussions. This material is based upon work supported by: (i) The NSFC under grant Nos. 11890712 and 12075129 and Guangdong Major Project of Basic and Applied Basic Research No. 2020B0301030008 (J.Z. and P.Z.); (ii) The Natural Sciences and Engineering Research Council of Canada (S.S.); (iii) The Fonds de recherche du Québec - Nature et technologies (FRQNT) through the Programmede Bourses d'Excellencepour Étudiants Étrangers (PBEEE) scholarship (S.S.); (iv) The BMBF funding under the ErUM-Data project and the AI grant at FIAS of SAMSON AG, Frankfurt (K.Z.); (v) The GPU Grant of the NVIDIA Corporation (K.Z.); (vi) The U.S. Department of Energy, Office of Science, Office of Nuclear Physics through the Contract No. DE-SC0012704 (S.M.); (vii) The U.S. Department of Energy, Office of Science, Office of Nuclear Physics and Office of Advanced Scientific Computing Research, within the framework of Scientific Discovery through Advance Computing (SciDAC) award Computing the Properties of Matter with Leadership Computing Resources (S.M.). 


\section{References}

[1] T. Matsui, H. Satz, Phys. Lett. B 178, 416 (1986)

[2] F. Karsch, M.T. Mehr, H. Satz, Z. Phys. C 37, 617 (1988)

[3] J.P. Blaizot, J.Y. Ollitrault, Phys. Rev. Lett. 77, 1703 (1996)

[4] P. Braun-Munzinger, J. Stachel, Phys. Lett. B 490, 196 (2000)

[5] S. Digal, P. Petreczky, H. Satz, Phys. Rev. D 64, 094015 (2001)

[6] L. Grandchamp, R. Rapp, G.E. Brown, Phys. Rev. Lett. 92, 212301 (2004)

[7] T. Song, K.C. Han, C.M. Ko, Phys. Rev. C 84, 034907 (2011)

[8] X. Du, R. Rapp, Nucl. Phys. A 943, 147 (2015)

[9] Y. Liu, B. Chen, N. Xu, P. Zhuang, Phys. Lett. B 697, 32 (2011)

[10] K. Zhou, N. Xu, Z. Xu, P. Zhuang, Phys. Rev. C 89, 054911 (2014)

[11] R. Katz, P.B. Gossiaux, Annals Phys. 368, 267 (2016)

[12] X. Yao, W. Ke, Y. Xu, S.A. Bass, B. Müller, JHEP 21, 046 (2020)

[13] A. Islam, M. Strickland, Phys. Lett. B 811, 135949 (2020)

[14] S. Chatrchyan et al. (CMS), Phys. Rev. Lett. 107, 052302 (2011)

[15] S. Chatrchyan et al. (CMS), Phys. Rev. Lett. 109, 222301 (2012), [Erratum: Phys.Rev.Lett. 120, 199903 (2018)]

[16] V. Khachatryan et al. (CMS), Phys. Lett. B 770, 357 (2017)

[17] A.M. Sirunyan et al. (CMS), Phys. Rev. Lett. 120, 142301 (2018)

[18] H. Satz, J. Phys. G 32, R25 (2006)

[19] J. Zhao, K. Zhou, S. Chen, P. Zhuang, Prog. Part. Nucl. Phys. 114, 103801 (2020)

[20] H.W. Crater, J.H. Yoon, C.Y. Wong, Phys. Rev. D 79, 034011 (2009)

[21] X. Guo, S. Shi, P. Zhuang, Phys. Lett. B 718, 143 (2012)

[22] M. Laine, O. Philipsen, P. Romatschke, M. Tassler, JHEP 03, 054 (2007)

[23] A. Beraudo, J.P. Blaizot, C. Ratti, Nucl. Phys. A 806, 312 (2008)

[24] N. Brambilla, J. Ghiglieri, A. Vairo, P. Petreczky, Phys. Rev. D 78, 014017 (2008)

[25] N. Brambilla, M.A. Escobedo, J. Ghiglieri, J. Soto, A. Vairo, JHEP 09, 038 (2010)

[26] A. Rothkopf, T. Hatsuda, S. Sasaki, Phys. Rev. Lett. 108, 162001 (2012)

[27] Y. Burnier, O. Kaczmarek, A. Rothkopf, Phys. Rev. Lett. 114, 082001 (2015)

[28] Y. Burnier, O. Kaczmarek, A. Rothkopf, JHEP 12, 101 (2015)

[29] D. Bala, S. Datta, Phys. Rev. D 101, 034507 (2020)

[30] R. Larsen, S. Meinel, S. Mukherjee, P. Petreczky, Phys. Rev. D 100, 074506 (2019)

[31] R. Larsen, S. Meinel, S. Mukherjee, P. Petreczky, Phys. Lett. B 800, 135119 (2020)

[32] R. Larsen, S. Meinel, S. Mukherjee, P. Petreczky, Phys. Rev. D 102, 114508 (2020)

[33] S. Shi, K. Zhou, J. Zhao, S. Mukherjee, P. Zhuang (2021), 2105.07862

[34] M. Leshno, S. Schocken, Neural Networks 6, 861 (1993)

[35] A. Kratsios, Annals of Mathematics and Artificial Intelligence 89, 435 (2021)

[36] M. Tanabashi et al. (Particle Data Group), Phys. Rev. D 98, 030001 (2018)

[37] D. Lafferty, A. Rothkopf, Phys. Rev. D 101, 056010 (2020)

[38] J.H. Weber, Complex static potential in lattice qcd (2021), https://indico.cern. ch/event/838970/contributions/4258563/

[39] R. Larsen, Excited states of bottomonia in qgp from lattice qcd (2021), https: //indico.cern.ch/event/985652/contributions/4302187/

[40] A. Mocsy, P. Petreczky, Phys. Rev. Lett. 99, 211602 (2007)

[41] M. Ali-Akbari, D. Giataganas, Z. Rezaei, Phys. Rev. D 90, 086001 (2014)

[42] L. Thakur, U. Kakade, B.K. Patra, Phys. Rev. D 89, 094020 (2014) 